\title{
The distribution of haemoglobin $C$ and its prevalence in newborns in Africa
}

SUBJECT AREAS: EPIDEMIOLOGY

POPULATION SCREENING

GENETICS RESEARCH

POPULATION GENETICS

Received

19 October 2011

Accepted

2 April 2013

Published

17 April 2013

Correspondence and requests for materials should be addressed to F.B.P. (fred.piel@zoo. ox.ac.uk)

\author{
Frédéric B. Piel',2, Rosalind E. Howes', Anand P. Patil', Oscar A. Nyangiri ${ }^{3}$, Peter W. Gething', \\ Samir Bhatt' ${ }^{1}$ Thomas N. Williams ${ }^{3,4}$, David J. Weatherall ${ }^{5} \&$ Simon I. Hay ${ }^{1}$
}

${ }^{1}$ Spatial Ecology and Epidemiology Group, Tinbergen Building, Department of Zoology, University of Oxford, South Parks Road, Oxford OXI 3PS, United Kingdom, ${ }^{2}$ Evolutionary Ecology of Infectious Disease Group, Tinbergen Building, Department of Zoology, University of Oxford, South Parks Road, Oxford OX1 3PS, United Kingdom, ${ }^{3}$ Kenya Medical Research Institute/Wellcome Trust Programme, Centre for Geographic Medicine Research-Coast, PO Box 230, Kilifi District Hospital, Kilifi, Kenya, ${ }^{4}$ Department of Medicine, Imperial College, St Mary's Hospital, Praed Street, London W21NY, United Kingdom, ${ }^{5}$ Weatherall Institute of Molecular Medicine, John Radcliffe Hospital, Oxford OX3 9DS, United Kingdom.

Haemoglobin $\mathrm{C}(\mathrm{HbC})$ is one of the commonest structural haemoglobin variants in human populations. Although $\mathrm{HbC}$ causes mild clinical complications, its diagnosis and genetic counselling are important to prevent inheritance with other haemoglobinopathies. Little is known about its contemporary distribution and the number of newborns affected. We assembled a global database of population surveys. We then used a Bayesian geostatistical model to create maps of $\mathrm{HbC}$ frequency across Africa and paired our predictions with high-resolution demographics to calculate heterozygous (AC) and homozygous (CC) newborn estimates and their associated uncertainty. Data were too sparse outside Africa for this methodology to be applied. The highest frequencies were found in West Africa but $\mathrm{HbC}$ was commonly found in other parts of the continent. The expected annual numbers of AC and CC newborns in Africa were 672,117 (interquartile range (IQR): 642,116-705,163) and 28,703 (IQR: 26,027-31,958), respectively. These numbers are about two times previous estimates.
( aemoglobin $\mathrm{C}(\mathrm{HbC})$ is a structural variant of normal haemoglobin $(\mathrm{HbA})$ caused by an amino acid substitution at position 6 of the $\beta$-globin chain $(\beta 6 \mathrm{Glu}-\mathrm{Lys})^{1}$. It is one of the most prevalent abnormal haemoglobin mutations globally alongside haemoglobin $\mathrm{S}$, which occurs at the same position (HbS; $\beta 6 \mathrm{Glu}-\mathrm{Val}$ ), and haemoglobin $\mathrm{E}$ (HbE, $\beta 26 \mathrm{Glu}-\mathrm{Lys})$. In $\mathrm{HbC}$ heterozygote individuals (AC), this trait is asymptomatic. Homozygosity (CC) causes clinically mild haemolytic anaemia, due to the reduced solubility of the red blood cells which can lead to crystal formation ${ }^{2}$. $\mathrm{HbC}$ is mainly of clinical significance when inherited in combination with HbS (sickle-haemoglobin C disease), causing chronic haemolytic anaemia and intermittent sickle cell crises, slightly less severe or frequent than in homozygous HbS patients (SS), and when co-inherited with $\beta$-thalassaemia (haemoglobin C- $\beta$ thalassaemia), causing moderate haemolytic anaemia with splenomegaly ${ }^{3}$.

$\mathrm{HbC}$ allele frequencies above $15 \%$ have been described in West African populations ${ }^{4}$. As for HbS, the selection pressure resulting from malaria protection has been suggested to explain the high prevalence of this polymorphism in a number of populations (commonly referred to as the malaria hypothesis) ${ }^{5,6}$. It has been found that $\mathrm{HbC}$ provides near full protection against Plasmodium falciparum malaria in homozygous (CC) individuals and intermediate protection in heterozygous $(\mathrm{AC})$ individuals ${ }^{7}$. Although these advantages (milder clinical severity and protection from severe and fatal Plasmodium falciparum malaria in both AC and CC individuals) could suggest that $\mathrm{HbC}$ has better fitness than $\mathrm{HbS}^{8,9}$, until the recent waves of human migration in the last few centuries, its distribution was limited to a much smaller geographic area than that of $\mathrm{HbS}^{5}$.

$\mathrm{HbC}$ is now widespread ${ }^{10-13}$, and it is widely assumed that $\mathrm{HbC}$ expanded to its current distribution from a unique origin in West Africa ${ }^{14-16}$, although an independent origin in southeast Asia has been suggested ${ }^{17,18}$. The current distribution of $\mathrm{HbC}$ is poorly documented ${ }^{4,19}$, yet this information is necessary to assess its contribution to the increasing public health and economic burden of the haemoglobinopathies ${ }^{20}$. Here, as part of our efforts to create an open access online database of selected inherited blood disorders and polymorphisms ${ }^{5,21-23}$, we have reviewed the published literature and assembled representative population survey data on $\mathrm{HbC}$ allele frequencies at the global scale. Following careful inclusion criteria and georeferencing of these data, this database formed the evidence-base for a Bayesian model-based geostatistical (MBG) framework ${ }^{24,25}$ which we developed to predict a continuous map of the distribution of $\mathrm{HbC}$ across Africa. Pairing these predictions with high resolution 
population data and national crude birth rates allowed the expected numbers of newborns affected annually by $\mathrm{HbC}$ trait (AC) and disease (CC) to be estimated.

\section{Results}

Database. Our searches identified 174 data sources (listed in Supplementary Information) with $\mathrm{HbC}$ data which allowed calculation of an allele frequency for representative population samples at specific locations. These included data for 445 spatially unique locations (Figure 1). The total number of individuals tested was $7,540,983$. Sample sizes ranged from four individuals to $3,212,374$. The mean sample size was 16,946 individuals. Some $82 \%$ of the population samples tested fewer than 1,000 individuals. Although $45 \%$ of the population surveys were conducted on the African continent, these represented only $5 \%$ of the total number of individuals examined. Our searches revealed that about half (51\%) of the total 1,992 references from the online searches on $\mathrm{HbC}$ found has been published after 1985, the publication year of Livingstone's latest database (Supplementary Figure $\mathrm{S} 1)^{4}$. About $60 \%$ of the surveys used for the present study pre-dated 1985. Amongst our 445 datapoints, an absence of $\mathrm{HbC}$ was reported in $48 \%$ of them. Few surveys $(\mathrm{n}=7)$ indicating null frequencies within West Africa (Figure 1) have been published. Allele frequencies above $20 \%$ were observed in the eastern (29\%) and western (24\%) parts of Burkina Faso. Apart from one survey in southern Ghana, frequencies above $10 \%$ were only observed across Burkina Faso and the adjacent northern parts of Ghana, Togo and Benin (32 surveys). Although $\mathrm{HbC}$ has been found in other parts of Africa (including Angola, Kenya, Egypt and Algeria), none of the surveys conducted in southern Cameroon (seven surveys) or southern Chad (three surveys) reported its presence. In North America, $\mathrm{HbC}$ was found both on the West and East coast of the United States (18 surveys), but was absent from eight of nine surveys in Mexico. In South America, $\mathrm{HbC}$ was identified in most surveys conducted in Brazil (33 surveys) and was commonly observed (14 out of 38 surveys) in Columbia, Venezuela and French Guiana. It was not observed in Peru, Bolivia or Chile (15 surveys). Ten of the 13 surveys conducted in the Caribbean islands reported the presence of $\mathrm{HbC}$. In Europe, $\mathrm{HbC}$ was observed in capital cities (London, Paris, Madrid, and Brussels) as well as in parts of Sardinia, but not in Greece or Albania. In the Middle East, surveys conducted on the eastern coast of Saudi Arabia, in eastern Iraq and along the Pakistani coast each reported a few cases. In Asia, none of the population surveys, including the micro-mapping survey conducted in Sri Lanka, found HbC. No surveys were available from Oceania.

Map. Our continuous map predicted $\mathrm{HbC}$ allele frequencies across Africa. The predicted posterior mean is presented in Figure 2. The maximum of the predicted posterior median $\mathrm{HbC}$ allele frequency was $16.0 \%$ (interquartile range (IQR): $12.0 \%-21.0 \%$ ) in the eastern part of Burkina Faso. Median frequencies above $12.5 \%$ were predicted around that area, as well as in north-eastern Ghana, northern Togo and north-western Benin. We predicted median frequencies above $7.5 \%$ in western Burkina Faso (up to $11.0 \%$ (IQR: $8.0 \%-14.0 \%$ ), remaining parts of northern Ghana and Benin, as well as across most of Mali, eastern Mauritania and southern Algeria. Median frequencies reached up to $5.0 \%$ in most other parts of western Africa, despite pockets of low frequencies (e.g. in Sierra Leone and Guinea-Bissau) and a sharp longitudinal decrease across Nigeria. The model also predicted a corridor of mean frequencies of about $1.0 \%$ between West Africa and Egypt, based on the finding of $\mathrm{HbC}$ in the few surveys conducted in these areas of low population density. Patches of median frequencies below $1 \%$ were predicted in Gabon, eastern Angola, and Uganda. The uncertainty associated with these predictions is shown in Figure 3. The IQR distribution reflects the distribution and heterogeneity of the data. It reaches values up to $11.0 \%$ (IQR: 9.0\%-20.0\%) in eastern Burkina Faso. The IQR is mostly above 5.0\% across Mali and northern Ghana, Togo and Benin where very few surveys were available.

National and regional estimates of affected newborns. We estimated that, in 2010, in Africa, 672,117 (IQR: 642,116-705,163) and 28,703 babies (IQR: 26,027-31,958) were born with the AC and CC genotypes respectively (Table 1 ). At the national scale, $56 \%$ of the AC newborns were expected to be from Burkina Faso (131,454 [IQR: 117,825-146,173]), Ghana (98,153 [IQR: 87,225-110,939]) and Nigeria (IQR: $148,423[112,961-197,818]$ ), and $76 \%$ of the CC newborns in these three countries (Burkina Faso: 9,592 [IQR: 7,258-13,259]); Ghana: 4,707 [IQR: 3,601-6,546]) and Nigeria: 3,099 [IQR: 1,822-5,948]) and Mali (4,354 [IQR: 2,257-9,952]).

Validation statistics. The mean error, mean absolute error and root mean square (RMS) error associated with our allele frequency predictions were $0.012+0.026,0.019+0.027$, and $0.026+0.037$, respectively. The overall bias of the predictions is thus very small, while their accuracy and precision can be considered as good. The

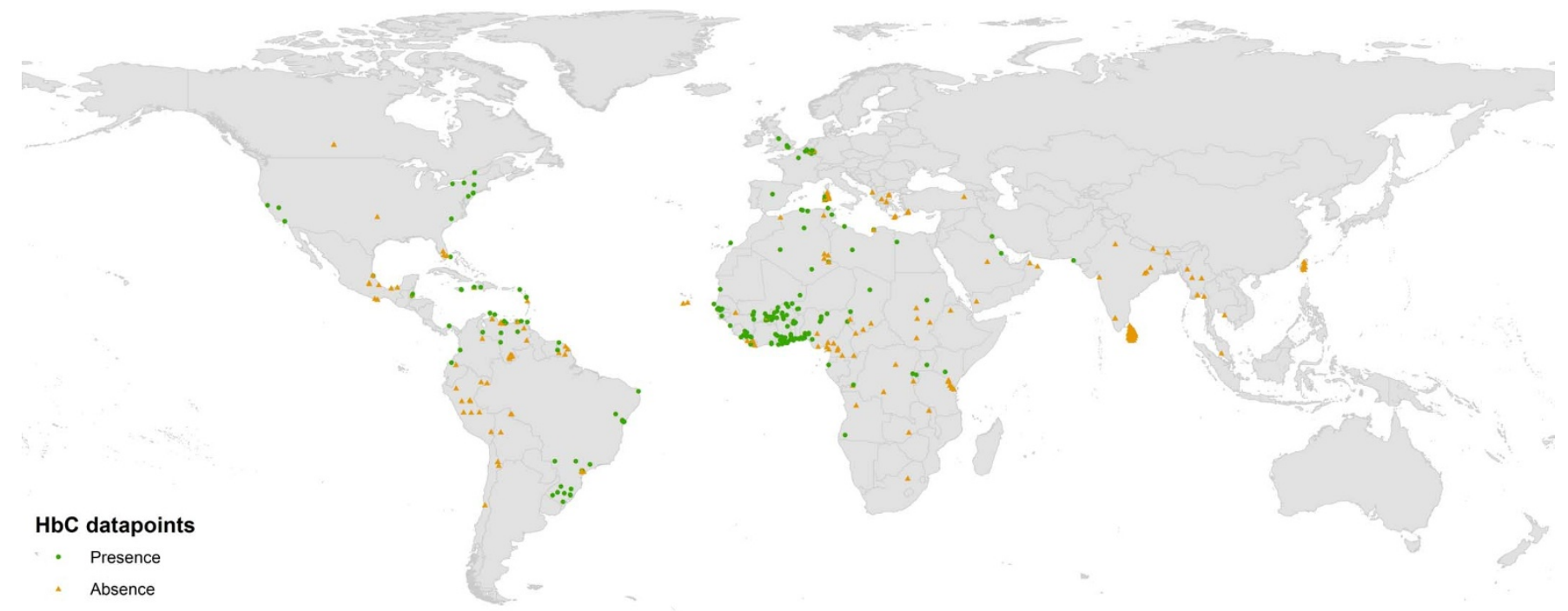

Figure 1 Global distribution of surveys on HbC. Green dots and orange triangles indicate surveys which found $\mathrm{HbC}$ to be present and absent from the population sample respectively. Created with ESRI ArcGIS 10.1. 


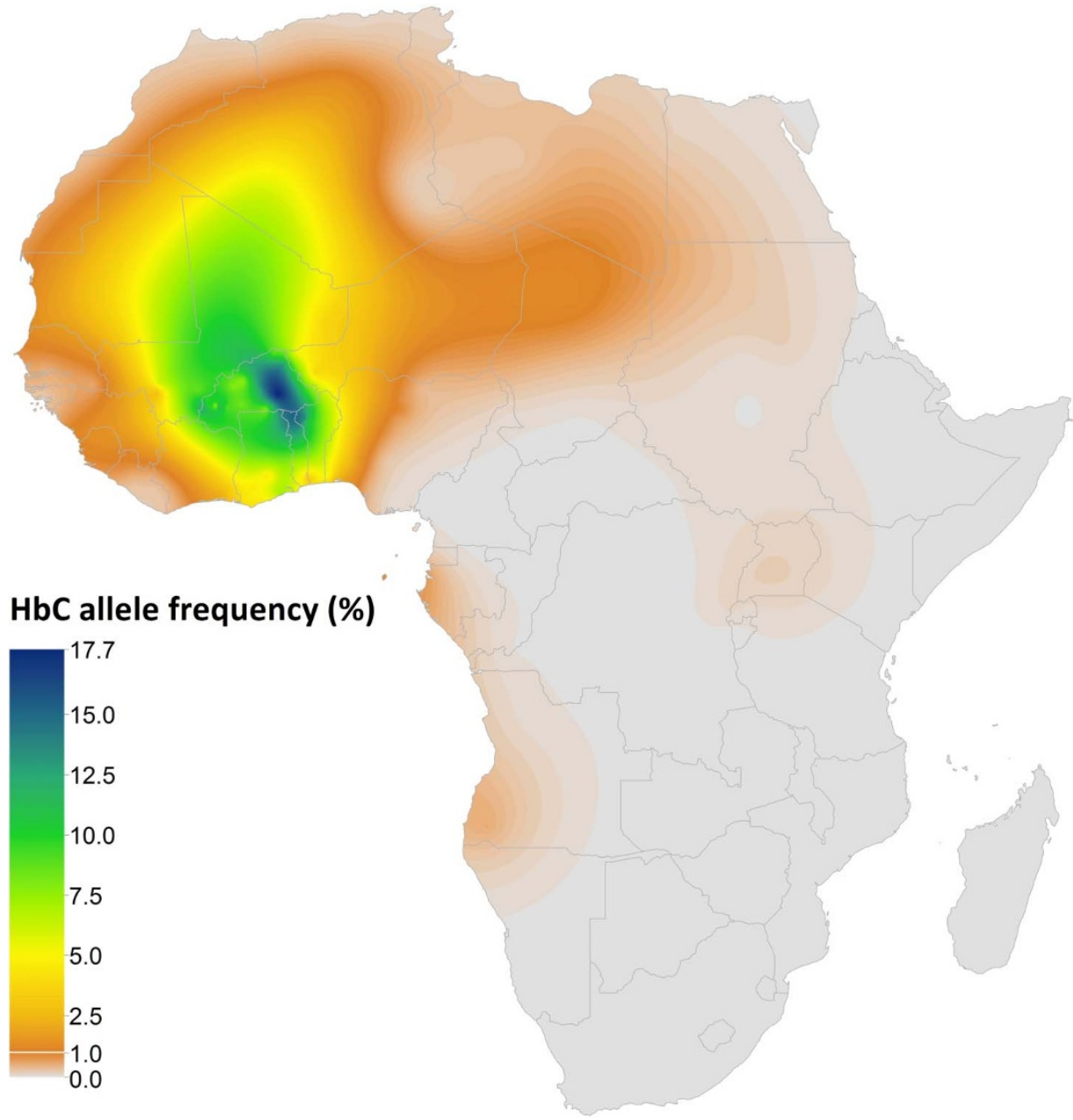

Figure $2 \mid$ Summary map of $\mathrm{HbC}$ predicted allele frequency in Africa. Raster map ( $5 \mathrm{~km} \times 5 \mathrm{~km}$ ) of HbC allele frequency (posterior mean) generated by a Bayesian model-based geostatistical framework. Created with ESRI ArcGIS 10.1.

Monte Carlo standard errors (SE) associated with the areal estimates at regional and national levels respectively are shown in Supplementary Table S1.

\section{Discussion}

It is expected that the global economic burden of the haemoglobinopathies on public health will increase over the coming decades ${ }^{20}$. In order to assess this burden and to track spatial and temporal changes, it is crucial to have a good knowledge of the distribution and number of individuals affected by these disorders. The map of surveys on $\mathrm{HbC}$ provides a summary of currently available data and highlights areas where further research is needed. The predicted allele frequency maps reflect the contemporary distribution of this disorder in Africa. The newborn estimates give us a more precise idea of the public health importance of $\mathrm{HbC}$. Each result is discussed in detail below.

The originally confined distribution of $\mathrm{HbC}$ was described as early as the mid-1950s $\mathrm{s}^{26}$ - only a few years after the first identification of this particular haemoglobin variant ${ }^{1}$. Nevertheless, cartographic refinements have been nearly absent since then. In 1967, Frank B. Livingstone published impressively detailed maps of abnormal haemoglobins, which included $\mathrm{HbC}$, but he did not publish equivalent maps in the updated version of his database in $1985^{27}$. Further, these maps were discontinuous, both spatially (i.e. mapping data as points but not predicting at unobserved locations) and quantitatively (i.e. using a categorical allele frequency scale). In 1994, Cavalli-Sforza et al created a continuous allele frequency map of $\mathrm{HbC}$ as part of their suite of genetic maps ${ }^{28}$. The aim of the present study was to incorporate the additional data collected over the last decades and the technological improvements in mapping and modelling methods $^{27-29}$, allowing to provide precision metrics for the first time.

The distribution of datapoints somehow summarises our current knowledge of $\mathrm{HbC}$. Although most of the surveys were conducted in West Africa, where the highest frequencies are expected, their sample sizes are usually limited. The presence of $\mathrm{HbC}$ in surveys in Brazil, the United States and European capitals for which data were available (e.g. London and Paris) reflect the presence of immigrants from West Africa in these communities. More detailed data from Belgium suggest that $\mathrm{HbC}$ carriers might also be identified in smaller cities (Boemer, pers. com.) but no surveys focussed on non-urban areas where $\mathrm{HbC}$ is likely to be absent due to lower levels of admixture. Because of the small number of surveys available in the Middle East and of the possible misidentification of $\mathrm{HbC}$ and $\mathrm{HbE}$ with commonly used electrophoretic methods ${ }^{3}$, the eastern limit of the distribution of $\mathrm{HbC}$ is unclear.

According to our input data and predicted maps, $\mathrm{HbC}$ reaches its highest predicted frequencies in the western part of Burkina Faso. Our model suggests that high frequencies ( $\square$ 7.5\%) might extend across Burkina Faso, the northern parts of Ghana, Togo and Benin, and across Mali, eastern Mauritania and the southern part of Algeria. In the absence of any data from this area this is only informed by the common presence of $\mathrm{HbC}$ in northern Africa. Conducting population surveys in this area, as well as in Côte d'Ivoire and northern Ghana, would help refine knowledge of the extent of the distribution of high $\mathrm{HbC}$ allele frequencies in West Africa. 


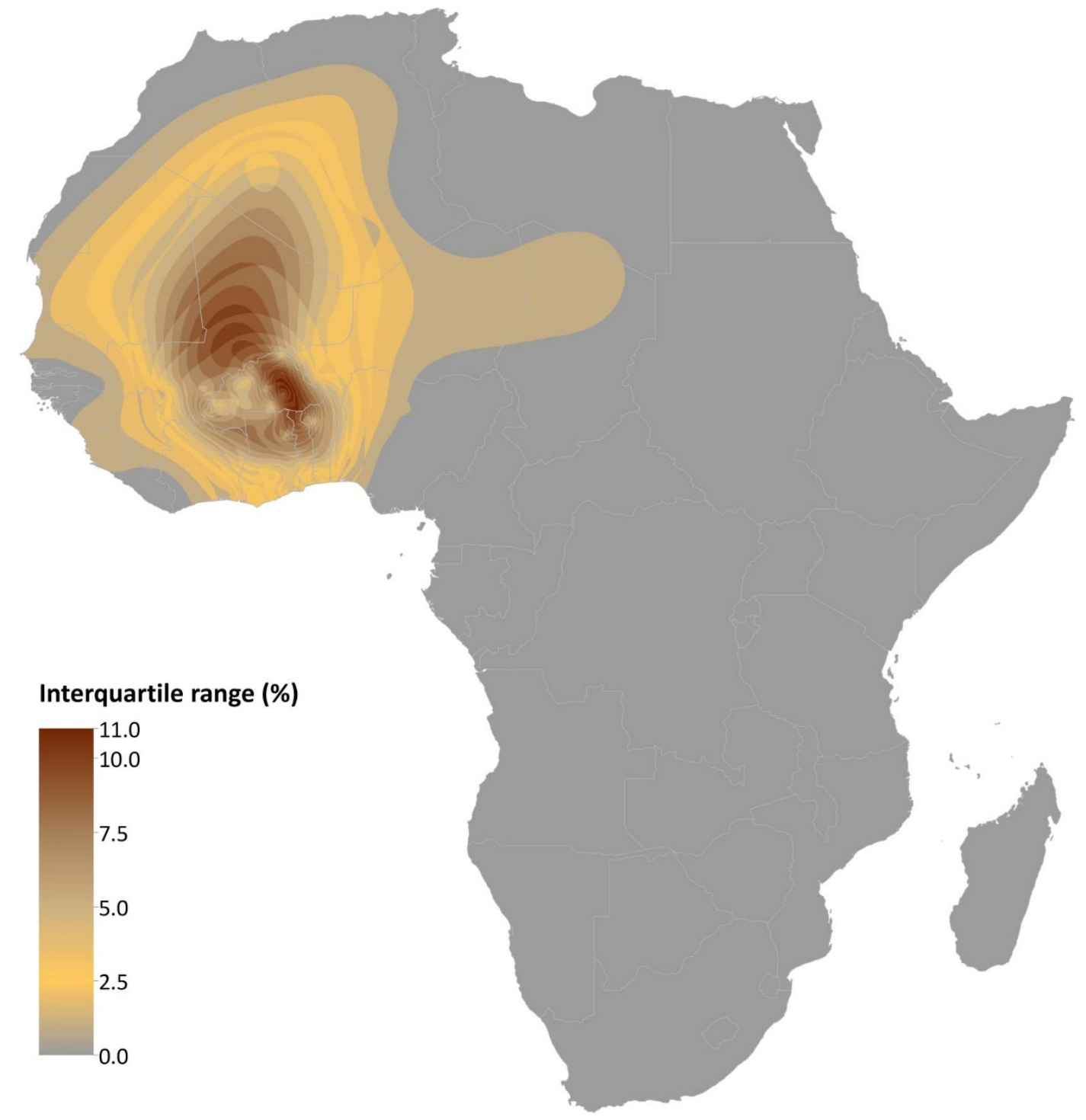

Figure 3 Uncertainty map in $\mathrm{HbC}$ predicted allele frequency in Africa. Interquartile range (50\% probability) of the per-pixel predicted allele frequency. Created with ESRI ArcGIS 10.1.

It is usually considered that the $\mathrm{HbS}$ mutation occurred at least twice in human history, once in Africa and once in $\mathrm{Asia}^{30-33}$; although several haplotypes have been identified for the $\mathrm{HbC}$ mutation, it is assumed to have a single origin in western Africa ${ }^{15,34}$. A recent case study conducted in Thailand suggested that one local haplotype might indicate an independent non-African origin of $\mathrm{HbC}^{18}$. The small number of cases identified so far, the absence of $\mathrm{HbC}$ in population surveys included in our database for India and Southeast Asia, and the absence of $\mathrm{HbC}$ in recent unpublished studies carried in Cambodia, Malaysia and South China (Fucharoen, pers. com.) tend to suggest that this haplotype would have a very limited distribution and low frequency. Furthermore, not a single case of $\mathrm{HbC}$ was identified during the micro-mapping work conducted in Sri Lanka (Weatherall, pers. com.). Further investigation into the hypothesis of an independent $\mathrm{HbC}$ mutation in Southeast Asia and its fitness in the presence of thalassaemias and haemoglobin $\mathrm{E}$ would provide a valuable contribution to our understanding of epistatic interactions between haemoglobinopathies ${ }^{35,36}$.

There is strong evidence for the protective effect of HbS against clinical Plasmodium falciparum malaria ${ }^{37}$. It is usually assumed that $\mathrm{HbC}$ also protects against malaria, but to a much lesser extent than $\mathrm{HbS}$ as reflected by its relatively limited original distribution ${ }^{8}$. An apparent inverse correlation between $\mathrm{HbS}$ and $\mathrm{HbC}$ allele frequencies in West Africa has been described ${ }^{8,33,38}$. The map presented here represents the contemporary distribution of $\mathrm{HbC}$ within Africa which because of human migration over the last few centuries is less useful than a map of pre-migration frequencies for investigating such a correlation ${ }^{5}$. Such maps and investigations are planned in future applications of this work. Furthermore, there is some evidence suggesting that other genes might also affect the level of malaria protection conveyed by $\mathrm{HbC}^{39}$.

Global, regional and national estimates of population affected represent important tools to assess the status of a particular disease, to follow its changes over space and time, and to guide associated public health policy. In 2008, Modell and Darlison published various estimates, including the proportion of pregnant women with $\mathrm{AC}$ and the number of CC conceptions, and derived service indicators for the haemoglobin disorders ${ }^{19}$. Here, we overcome several methodological limitations in order to provide updated newborn estimates. First, we performed online searches covering the 1950-2010 period to include recent data and selected data based on strict inclusion criteria to exclude non-representative surveys. Second, each survey was georeferenced as precisely as possible, enabling better representation of spatial heterogeneity. Third, we used high-resolution population 


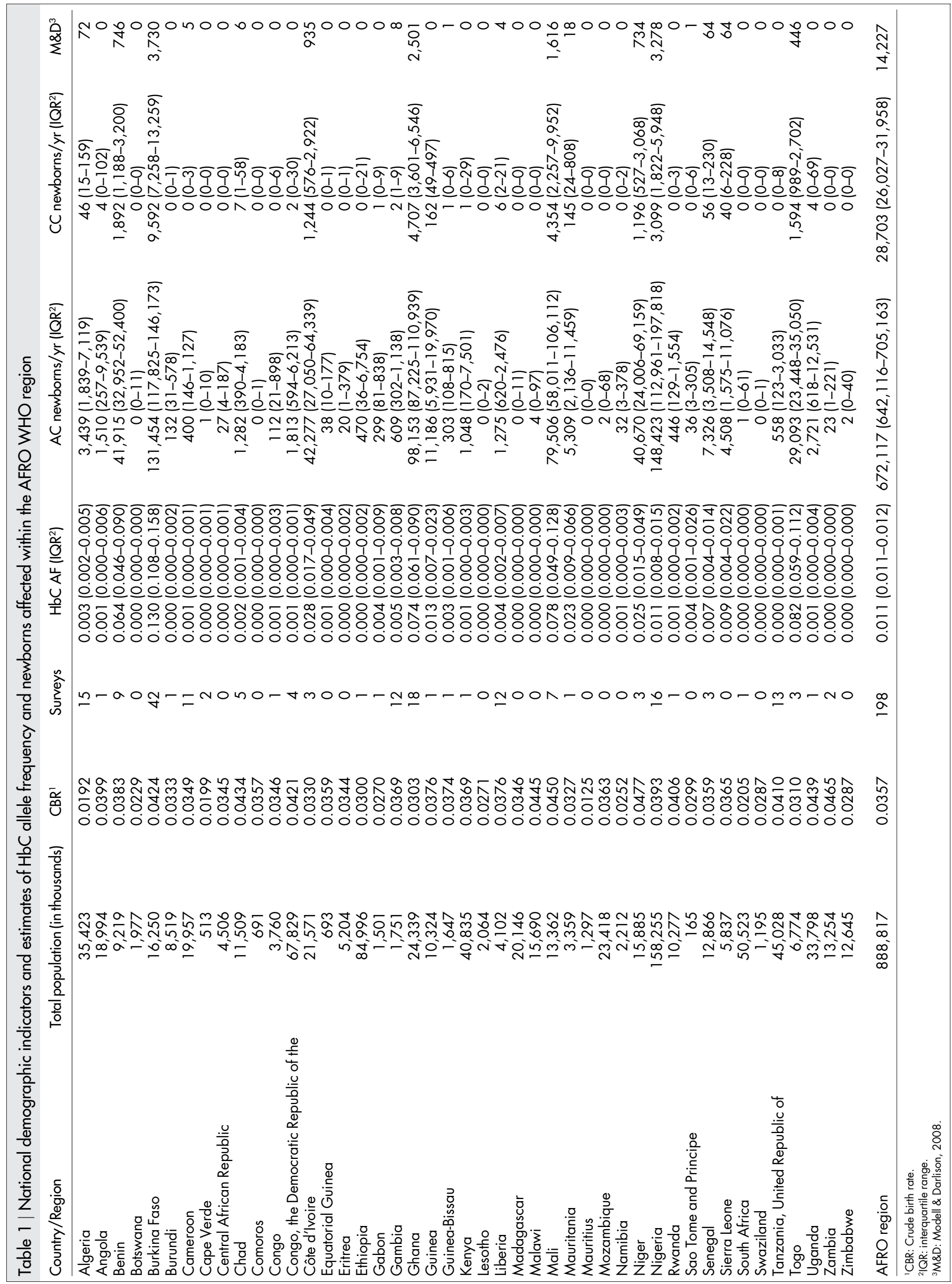


distribution data to relate these heterogeneities with the distribution of human populations. Finally, we calculated our predictions within a Bayesian MBG framework, which allowed calculation of the precision associated with our estimates.

Using 2003 UN demographics, Modell and Darlison estimated a global total of 14,719 CC newborns including 14,227 (97\%) in the AFRO region (Table 1). Their estimates were conservative (i.e. minimum figures) and estimates for countries where no $\mathrm{HbC}$ data were available were set to zero (e.g. Kenya or the Democratic Republic of the Congo). No estimates were published for the AC newborns and the precision of the estimates published was unknown. Our regional estimate for CC newborns in 2010 is about twofold higher, at 28,703 newborns $(\times 1.8$ for the $25 \%$-quartile estimate and $\times 2.2$ for the $75 \%$ quartile estimate). This large difference is mostly due to higher predictions in West African countries. Because of high heterogeneity in $\mathrm{HbC}$ allele frequency, extrapolating an average allele frequency to a national population can lead to an underestimation (or overestimation) of the number of individuals affected. In Burkina Faso, most of the survey data come from the western part where frequencies are comparatively lower than in the eastern part of the country. Similarly, there is very little data from the northern parts of Ghana, Togo and Benin - countries in which $\mathrm{HbC}$ allele frequencies tend to gradually increase across a latitudinal gradient (Ohene-Frempong, pers. com.). Not accounting for the distribution of the surveys within West African countries or their population distribution (i.e. high population density in areas of low $\mathrm{HbC}$ frequency and low population density in areas of high frequency will produce dramatically different estimates compared to the reverse situation) could therefore result in underestimating the number of newborns affected.

Although $\mathrm{HbC}$ causes only relatively mild clinical complications in AC and CC individuals, a good knowledge of its distribution and allele frequencies represents a useful tool to better assess its contribution,

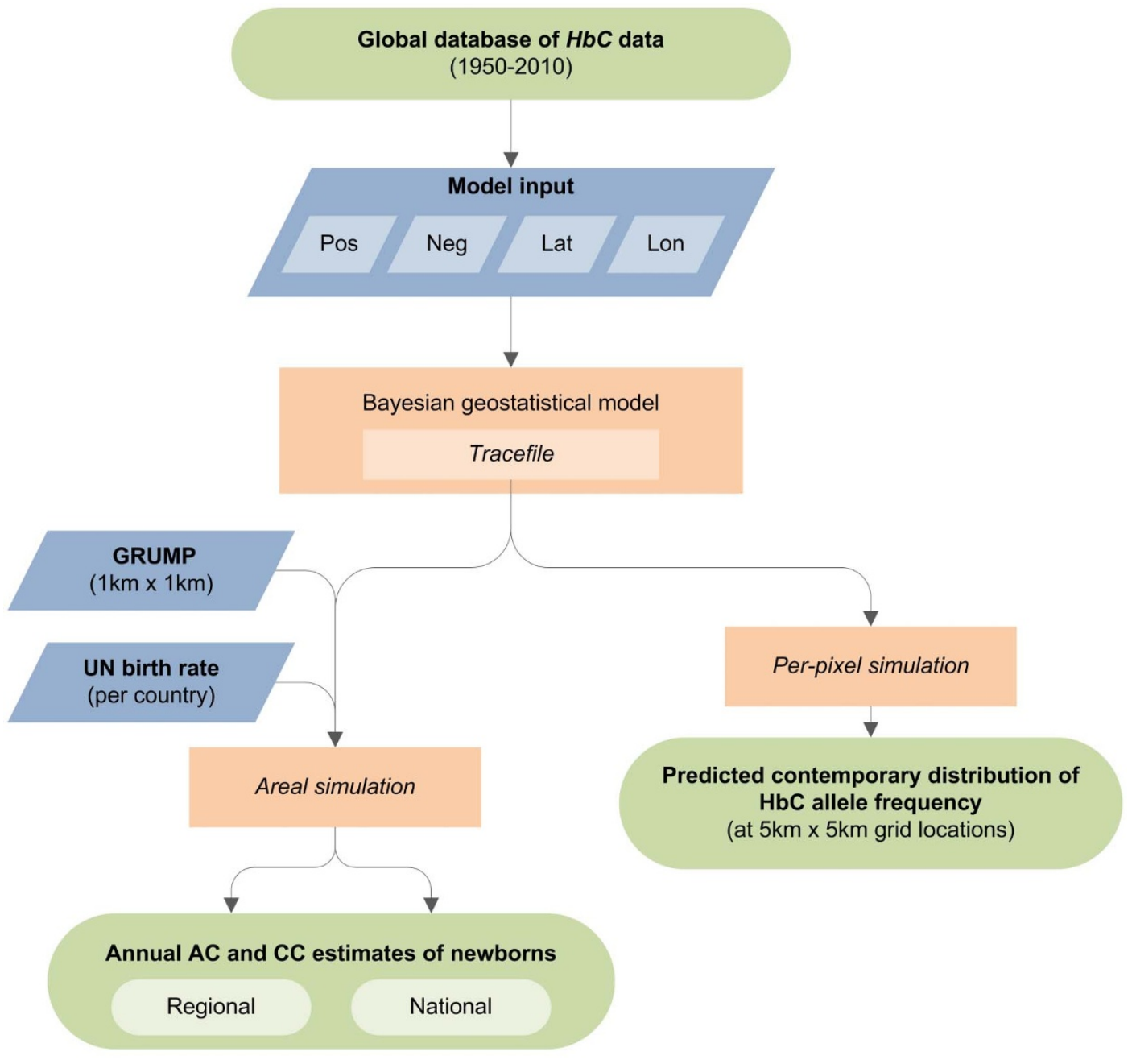

Legend

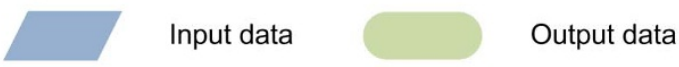

Model and experimental procedures

Pos: number of $\mathrm{C}$ alleles in the population sample.

Neg: number of non-C alleles in the population sample.

Lat: Latitude.

Lon: Longitude.

UN: United Nations; GRUMP: Global rural-urban mapping project

Figure $4 \mid$ Schematic overview of the approach. Blue diamonds describe input data. Orange boxes denote models and experimental procedures. Green rods indicate output data. Created with Microsoft Office Visio 2007. 
through compound individuals with $\mathrm{HbSC}$ disease or $\mathrm{HbC} / \beta$-thalassaemia, to the global burden of the haemoglobinopathies ${ }^{20,40}$. A bespoke multi-allelic model, ideally using an age-correction based on the mortality of SC individuals and accounting for deviation from Hardy-Weinberg assumptions $s^{41,42}$, would be required to estimate the number of SC newborns within a similar framework. The availability of the map and estimates presented here for $\mathrm{HbC}$, alongside similar products developed for $\mathrm{HbS}^{22}$, will facilitate such a study. The current lack of an updated database on the thalassaemias makes the calculation of the number of $\mathrm{HbC} / \beta$-thalassaemia compound newborns currently difficult. These various limitations will be the focus of future work.

Globally, our database suggests that human migrations are probably the main drivers shaping the contemporary distribution of the haemoglobinopathies, exerting a greater influence than positive selection driven by protection against malaria, which seems to have been the main factor in the past ${ }^{5,33}$. Although selection is likely to still influence the distribution of haemoglobinopathies in malarious regions, this change could contribute to shaping their future distributions, particularly in the context of epidemiological transition ${ }^{20}$ and malaria elimination ${ }^{43}$ efforts. The work presented here could not fully reflect those changes in the distribution of $\mathrm{HbC}$ due to the relative paucity of data outside of Africa. Nevertheless, our map provides a unique picture of the current distribution of $\mathrm{HbC}$ in Africa, while our estimates suggest that the annual number of $\mathrm{AC}$ and CC newborns might have been largely underestimated previously. In the long term, additional data will allow creating a global contemporary map and calculating global estimates and the development of a multi-allelic model would allow the calculation of similar estimates for SC and C- $\beta$ Thal compound individuals, which have a greater impact on clinical burden. This forms part of our plans for further work on the haemoglobinopathies.

\section{Methods}

A schematic overview of the methods used is provided as Figure 4. The methodology is briefly described below. Further details are available in the Supplementary Information.

Data sources. To identify publications with $\mathrm{HbC}$ allele frequency data, we undertook a comprehensive online data search using PubMed ${ }^{44}$, ISI Web of Knowledge ${ }^{45}$, and Scopus ${ }^{46}$ bibliographic databases. The following keywords were used: 'haemoglobin C' and 'hemoglobin C'. Searches performed on May 3, 2011 returned 1,275, 558 and 1,827 references in PubMed, ISI Web of Science and Scopus respectively. After duplicate removal, we identified 1,992 unique references which were then reviewed according to inclusion criteria, the main ones being that: i) the source included primary data on $\mathrm{HbC}$ frequency; ii) the population samples were representative of the local communities (data from targeted screening or selected population samples, such as Afro-Americans, were excluded) and iii) the survey location could be georeferenced precisely ${ }^{5}$. Additional data from unpublished sources fulfilling these criteria (particularly from the MalariaGEN Consortium) ${ }^{47}$ were also included in the global database. When several surveys conducted at the same location met the inclusion criteria, only the most representative one (based on a combination of criteria including the year of the survey, the sample size and the diagnostic method) was used. The list of data sources used is shown in the Supplementary Information and the database is freely available online (http://www.map.ox.ac.uk).

Bayesian model-based geostatistical (MBG) framework. Numbers of A (neg) and C (pos) alleles, based on the number of AA, AC and CC individuals found in each population survey conducted on the African continent were used as input to the model alongside the surveys' geographic coordinates (latitude and longitude). For studies reporting the absence of any haemoglobin variants, all individuals were considered as AA. The model generated two distinct types of output across Africa: estimates of $\mathrm{HbC}$ allele frequency for every $5 \times 5 \mathrm{~km}$ pixel (i.e. estimates at point locations), and estimates of AC and CC newborns within each African country (i.e. estimates over areal units). In both cases, the full posterior predictive distribution $(\mathrm{PPD})^{24}$, was generated for the target quantity using 500,000 Markov chain Monte Carlo (MCMC) iterations ${ }^{48}$. A complete description of the model is given in the Supplementary Information.

Predicted distribution map in Africa. We assumed Hardy-Weinberg equilibrium for the calculation of $\mathrm{AC}$ and $\mathrm{CC}$ individuals from the $\mathrm{HbC}$ predicted allele frequency ${ }^{5,24}$. The mean and interquartile range (IQR; interval between the $25 \%$ and $75 \%$ percentiles of the PPD) maps were used to summarise the predictions and their associated uncertainty, respectively, for each pixel in African countries.
Estimates of newborns affected in Africa. The AC and CC predicted frequencies were weighted by i) high resolution $(1 \times 1 \mathrm{~km})$ population data from the 2010 adjusted beta version of the Global Rural Urban Mapping Project (GRUMP) ${ }^{49}$, and ii) national crude birth rates for 2010, derived from the 2010 revision online population database of the United Nations (UN) world population prospects ${ }^{50}$ (see Supplementary Information). To allow assessment of uncertainty measures associated with these aggregated population numbers, estimates were calculated using sampling from the whole predictive distributions of areal integrals (not just the mean summary map) within the area considered ${ }^{22,24,51}$. Areal estimates were calculated independently for the regional and national predictions. Summary estimates presented here include the median and IQR of the population predictions.

Model validation. Validation metrics were calculated by comparing the observed allele frequency for a $10 \%$ random hold-out sample of the African subset with the prediction output created from the remaining $90 \%$ of the African data $^{5}$. The validation metrics were summarised by i) the mean error, which indicates the average distance between the actual data points and the predicted values; ii) the mean absolute error, which measures the average magnitude of the errors in the predicted values; and iii) the root mean square (RMS) error ${ }^{29}$. These errors provide a measure of the model's overall bias, overall accuracy and overall precision, respectively. In order to calculate the Monte Carlo standard error (SE) ${ }^{52}$ associated with the newborn estimates, the areal calculations were repeated ten times at each scale (see Supplementary Information).

1. Itano, H. A. \& Neel, J. V. A new inherited abnormality of human hemoglobin. Proceedings of the National Academy of Sciences of the United States of America 36, 613-617 (1950).

2. Charache, S., Conley, C. L., Waugh, D. F., Ugoretz, R. J. \& Spurrell, J. R. Pathogenesis of hemolytic anemia in homozygous hemoglobin $\mathrm{C}$ disease. The Journal of Clinical Investigation 46, 1795-1811, doi:10.1172/jci105670 (1967).

3. Bain, B. J. Haemoglobinopathy Diagnosis. Second edn, (Blackwell Publishing Ltd, 2006).

4. Livingstone, F. B. Frequencies of Hemoglobin Variants: Thalassemia, the Glucose6-Phosphate Dehydrogenase Deficiency, G6Pd Variants and Ovalocytosis in Human Populations. (Oxford University Press, 1985).

5. Piel, F. B. et al. Global distribution of the sickle cell gene and geographical confirmation of the malaria hypothesis. Nature Communications 1, 104 (2010).

6. Haldane, J. B. S. The rate of mutation of human genes. In: Proceedings of the Eigth International Congress of Genetics. Hereditas 35, 267-273 (1949).

7. Modiano, D. et al. Haemoglobin C protects against clinical Plasmodium falciparum malaria. Nature 414, 305-308, doi:10.1038/35104556 (2001).

8. Modiano, D. et al. Haemoglobin S and haemoglobin C: 'quick but costly' versus 'slow but gratis' genetic adaptations to Plasmodium falciparum malaria. Human Molecular Genetics 17, 789-799, doi:10.1093/hmg/ddm350 (2008).

9. Hedrick, P. Estimation of relative fitnesses from relative risk data and the predicted future of haemoglobin alleles S and C. Journal of Evolutionary Biology 17, 221-224, doi:10.1046/j.1420-9101.2003.00635.x (2004).

10. van Heyningen, A. M. et al. Estimated incidence of sickle-cell disease in Aruba and St. Maarten suggests cost-effectiveness of a universal screening programme for St. Maarten. West Indian Med J 58, 301-304 (2009).

11. Olson, J. F., Ware, R. E., Schultz, W. H. \& Kinney, T. R. Hemoglobin C disease in infancy and childhood. Journal of Pediatrics 125, 745-747 (1994).

12. Gulbis, B. et al. Neonatal haemoglobinopathy screening in Belgium. J Clin Pathol 62, 49-52, doi:10.1136/jcp.2008.060517 (2009).

13. Patel, D. K., Patel, S., Mashon, R. S., Dash, P. M. \& Mukherjee, M. B. Diverse phenotypic expression of sickle cell hemoglobin $\mathrm{C}$ disease in an Indian family. Ann Hematol 90, 357-358, doi:10.1007/s00277-010-1014-1 (2011).

14. Talacki, C. A., Rappaport, E., Schwartz, E., Surrey, S. \& Ballas, S. K. Beta-globin gene cluster haplotypes in Hb C heterozygotes. Hemoglobin 14, 229-240 (1990)

15. Boehm, C. D., Dowling, C. E., Antonarakis, S. E., Honig, G. R. \& Kazazian, H. H., Jr. Evidence supporting a single origin of the beta(C)-globin gene in blacks. Am J Hum Genet 37, 771-777 (1985).

16. Kan, Y. W. \& Dozy, A. M. Evolution of the hemoglobin S and C genes in world populations. Science 209, 388-391 (1980).

17. Siriboon, W., Srisomsap, C., Winichagoon, P., Fucharoen, S. \& Svasti, J. Identification of HB C [beta(A3)GLU->LYS] in a Thai male. Hemoglobin 17, 419-425 (1993).

18. Sanchaisuriya, K. et al. Molecular characterization of hemoglobin C in Thailand. American Journal of Hematology 67, 189-193, doi:10.1002/ajh.1105 (2001).

19. Modell, B. \& Darlison, M. Global epidemiology of haemoglobin disorders and derived service indicators. Bulletin of the World Health Organization 86, 480-487, doi:S0042-96862008000600017 (2008).

20. Weatherall, D. J. The inherited diseases of hemoglobin are an emerging global health burden. Blood 115, 4331-4336, doi:10.1182/blood-2010-01-251348 (2010).

21. Howes, R. E. et al. The global distribution of the Duffy blood group. Nature Communications 2, 266, doi:10.1038/ncomms1265 (2011)

22. Piel, F. B. et al. Global epidemiology of sickle haemoglobin in neonates: a contemporary geostatistical model-based map and population estimates. Lancet 381, 142-151, doi:10.1016/S0140-6736(12)61229-X (2013). 
23. Howes, R. E. et al. G6PD deficiency prevalence and estimates of affected populations in malaria endemic countries: a geostatistical model-based map. PLoS Med 9, e1001339, doi:10.1371/journal.pmed.1001339 (2012).

24. Patil, A. P., Gething, P. W., Piel, F. B. \& Hay, S. I. Bayesian geostatistics in health cartography: the perspective of malaria. Trends in Parasitology 27, 246-253 (2011).

25. Patil, A. P., Huard, D. \& Fonnesbeck, C. J. PyMC: Bayesian stochastic modelling in Python. Journal of Statistical Software 35, 1-81 (2010).

26. Edington, G. M. \& Lehmann, H. The Distribution of Haemoglobin C in West Africa. Man 56, 34-36 (1956).

27. Livingstone, F. B. Abnormal hemoglobins in human populations: a summary and interpretation. (Aldine Publishing Company, 1967).

28. Cavalli-Sforza, L. L., Menozzi, P. \& Piazza, A. The History and Geography of Human Genes. (Princeton University Press, 1994).

29. Mayer, D. G. \& Butler, D. G. Statistical validation. Ecological Modelling 68, 21-32, doi:10.1016/0304-3800(93)90105-2 (1993).

30. Pagnier, J. et al. Evidence for the multicentric origin of the sickle cell hemoglobin gene in Africa. Proceedings of the National Academy of Sciences of the United States of America 81, 1771-1773 (1984).

31. Kulozik, A. E. et al. Geographical survey of beta S-globin gene haplotypes: evidence for an independent Asian origin of the sickle-cell mutation. Am J Hum Genet 39, 239-244 (1986).

32. Oner, C. et al. Beta S haplotypes in various world populations. Human Genetics 89 , 99-104 (1992).

33. Flint, J., Harding, R. M., Boyce, A. J. \& Clegg, J. B. The population genetics of the haemoglobinopathies. Baillière's Clinical Haematology 6, 215-262, doi: 10.1016/ s0950-3536(05)80071-x (1993).

34. Travi, M. et al. Molecular characterization of hemoglobin C in Sicily. American Journal of Hematology 39, 5-8 (1992).

35. Penman, B. S., Pybus, O. G., Weatherall, D. J. \& Gupta, S. Epistatic interactions between genetic disorders of hemoglobin can explain why the sickle-cell gene is uncommon in the Mediterranean. Proceedings of the National Academy of Sciences of the United States of America 106, 21242-21246 (2009).

36. Williams, T. N. et al. Negative epistasis between the malaria-protective effects of alpha +-thalassemia and the sickle cell trait. Nature Genetics 37, 1253-1257, doi:10.1038/ng1660 (2005).

37. Hill, A. V. S. et al. Common West African HLA antigens are associated with protection from severe malaria. Nature 352, 595-600 (1991).

38. Kreuels, B. et al. Differing effects of $\mathrm{HbS}$ and $\mathrm{HbC}$ traits on uncomplicated falciparum malaria, anemia, and child growth. Blood 115, 4551-4558, doi:10.1182/blood-2009-09-241844 (2010).

39. Atkinson, A., Barbier, M., Afridi, S., Fumoux, F. \& Rihet, P. Evidence for epistasis between hemoglobin $\mathrm{C}$ and immune genes in human Plasmodium falciparum malaria: a family study in Burkina Faso. Genes Immun (2011).

40. Weatherall, D., Akinyanju, O., Fucharoen, S., Olivieri, N. \& Musgrove, P. in Disease Control Priorities in Developing Countries Ch. 34, 663-680 (Oxford University Press, 2006)

41. Hardy, G. H. Mendelian proportions in a mixed population. Science 28, 49-50 (1908).

42. Weinberg, W. Über den Nachweis der Vererbung beim Menschen. Jahreshefte des Vereins für Vaterländische Naturkunde in Württemberg 64, 369-382 (1908).

43. Tatem, A. J. et al. Ranking of elimination feasibility between malaria-endemic countries. Lancet 376, 1579-1591 (2010).

44. Pubmed. http://www.pubmed.gov (Accessed: 3 May 2011).

45. ISI Web of Knowledge. http://isiwebofknowledge.com (Accessed: 3 May 2011).

46. Scopus. http://www.scopus.com (Accessed: 3 May 2011).
47. Malaria Genomic Epidemiology Network Consortium. A global network for investigating the genomic epidemiology of malaria. Nature 456, 732-737 (2008).

48. Gilks, W. R., Richardson, S. \& Spiegelhalter, D. Markov chain Monte Carlo in Practice. (Chapman \& Hall/CRC, 1995).

49. Balk, D. L. et al. Determining global population distribution: methods, applications and data. Advances in Parasitology 62, 119-156 (2006).

50. United Nations world population prospects: The 2010 Revision. http://esa.un.org/ unpp (Accessed: 25 July 2011).

51. Gething, P. W., Patil, A. P. \& Hay, S. I. Quantifying aggregated uncertainty in Plasmodium falciparum malaria prevalence and populations at risk via efficient space-time geostatistical joint simulation. Public Library of Science Computational Biology 6, e1000724 (2010).

52. Moran, P. A. P. The estimation of standard errors in Monte Carlo simulation experiments. Biometrika 62, 1-4, doi:10.1093/biomet/62.1.1 (1975).

\section{Acknowledgements}

The authors are grateful to the Technical Advisory Group (TAG) associated to this project. The members of the TAG, chaired by DJW, included Kevin Baird, Suthat Fucharoen, Dominic Kwiatkowski, Julie Makani, Kevin Marsh, Kirk Rockett, Graham Serjeant and Bill Wood. They also thank Kevin Baird, Sunetra Gupta, Catherine Moyes, and Bridget Penman for providing comments on the manuscript. Data from the MalariaGEN Consortium (http://www.malariagen.net) have been shared for inclusion in the database, and the authors acknowledge all the Consortium contributing collaborators and members for collecting, preparing and genotyping the samples. FBP, REH and OAN were funded by a Biomedical Resources Grant (\#085406) from the Wellcome Trust (to SIH). SIH is funded by a Senior Research Fellowship (\#095066) from the Wellcome Trust that also supported PWG. APP was funded by a Biomedical Resources Grant from the Wellcome Trust (\#091835). TNW is funded by a Senior Clinical Fellowship (\#091758) from the Wellcome Trust. DJW is funded by the Wellcome Trust and acknowledges support from the March of Dimes, and the Anthony Cerami and Ann Dunne Foundation for World Health. The authors are also grateful for support from the Philippe Wiener - Maurice Anspach Foundation and the European Research Council (grant DIVERSITY). This paper was published with the permission of the director of KEMRI.

\section{Author contributions}

F.B.P. assembled the data, conceived the study and wrote the first draft of the manuscript with S.I.H.; O.A.N. and R.E.H. helped assemble and abstract the data; A.P.P. and P.W.G. conceived and helped implement the modelling and all computational tasks. All authors contributed to the revision of the final manuscript. The authors declare no competing financial interests. This work forms part of the output of the Malaria Atlas Project (MAP, http://www.map.ox.ac.uk), principally funded by the Wellcome Trust, U.K.

\section{Additional information}

Supplementary information accompanies this paper at http://www.nature.com/ scientificreports

Competing financial interests: The authors declare no competing financial interests.

License: This work is licensed under a Creative Commons Attribution 3.0 Unported License. To view a copy of this license, visit http://creativecommons.org/licenses/by/3.0/

How to cite this article: Piel, F.B. et al. The distribution of haemoglobin C and its prevalence in newborns in Africa. Sci. Rep. 3, 1671; DOI:10.1038/srep01671 (2013). 